\title{
Compared with Monocyte to High-Density Lipoprotein Ratio (MHR) and Neutrophil to Lymphocyte Ratio (NLR),Neutrophil to High-Density Lipoprotein Ratio (NHR) is more Valuable in the Inflammatory Process of Parkinson's Disease
}

Qingli Fan (D 1628018509@qq.com )

Qinghai Provincial People's Hospital

Zhu Liu

Qinghai University

Shizheng Wu

Qinghai Provincial People's Hospital

Yancheng Lei

Qinghai Provincial People's Hospital

Research

Keywords: Parkinson's disease, Neutrophil to high-density lipoprotein ratio, Neutrophil to lymphocyte ratio, Monocyte to high-density lipoprotein ratio, Course of disease, Severity

Posted Date: February 1st, 2021

DOl: https://doi.org/10.21203/rs.3.rs-185056/v1

License: (c) (1) This work is licensed under a Creative Commons Attribution 4.0 International License.

Read Full License

Version of Record: A version of this preprint was published at Lipids in Health and Disease on April 19th, 2021. See the published version at https://doi.org/10.1186/s12944-021-01462-4. 


\section{Abstract}

Background: Inflammatory response plays an important role in the pathologic process and prognosis of Parkinson's disease (PD).We investigated the relationship between the neutrophil to high-density lipoprotein ratio (NHR), neutrophil to lymphocyte ratio (NLR), and monocyte to high-density lipoprotein ratio (MHR) on the prediction of PD and its course and severity.

Methods: Patients with Parkinson's disease were selected $(n=101)$ and divided into three groups according to the onset cycle: $<6$ years $(n=64), 6-10$ years $(n=23)$ and $>10$ years $(n=14)$. And according to Hoehn and Yahr classification: 1 2.5 is the early stage $(n=55)$;Grades $3 \sim 5$ are divided into two groups $(n=46)$.In addition, healthy subjects $(n=97)$ matched with the above pd patients in the same period were selected as the control group.In this way, the influence of NHR, NLR, MHR and other indicators on corresponding groups is evaluated.

Results: Neutrophils, NHR and NLR in PD group were significantly higher than those in control group. nevertheless, lymphocyte, total cholesterol, low density lipoprotein and hemoglobin were significantly lower than those in the control group. Multi-factor logistic regression analysis indicated that NHR (odds ratio $(\mathrm{OR})=1.456,95 \% \mathrm{Cl}: 1.007 \sim 2.104, P=0.046)$ and $\mathrm{NLR}(\mathrm{OR}=1.663,95 \% \mathrm{Cl}: 1.101 \sim 2.513, P=0.016)$ were risk factors for Parkinson's disease, while MHR had no significant correlation with Parkinson's disease. The AUC(area under the ROC curve) of PD predicted by NHR and NLR were $0.648(95 \% \mathrm{Cl}: 0.572 \sim 0.724, P=0.0003)$ and $0.718(95 \% \mathrm{Cl}: 0.646 \sim 0.790, P<0.0001)$,respectively, and the critical values for optimal diagnosis were $3.104 \times 10^{9} / \mathrm{mmol}$ and $1.939 \times 10^{9} / \mathrm{mmol}$. Spearman analysis showed that NHR was significantly negatively correlated with the course of disease.

Conclusions: In summary, NHR not only has strong predictive value for PD disease, but also is closely related to the course of disease. NHR levels may be better predictors of long-term clinical outcomes in PD patients than MHR and NLR.

\section{Introduction}

Parkinson's disease (PD) is a common neurodegenerative disease. The global prevalence rate increases with the increase of age and increases faster than other neurological diseases,and the aging of the population also further promotes the occurrence of PD to a large extent ${ }^{[1]}$.Although the pathogenesis of Parkinson's disease is still unclear,inflammation has been proved to be involved in the pathogenesis and progression of Parkinson's disease ${ }^{[2-4]}$. Microglial cell (MG) hyperplasia in the brain is the pathophysiological basis of PD.MG secretes high levels of proinflammatory mediators,damages neurons,further activates microglial cells, and promotes inflammatory response ${ }^{[5]}$. Neutrophils,monocytes and lymphocytes,as components of white blood cells in circulating blood,increase in peripheral inflammation and participate in the development of clinical symptoms in PD patients ${ }^{[2,6-7]}$. The antiinflammatory and antioxidant effects of HDL-C(high-density lipoprotein cholesterol) have been well known, and the decreased levels of HDL-C are associated with systemic inflammation and endothelial 
cell dysfunction, thus participating in the progression of PD patients ${ }^{[4,8]}$.Some studies have found that neutrophil to lymphocyte ratio (NLR), neutrophil to HDL-C ratio (NHR), and monocyte to high-density lipoprotein cholesterol (HDL-C) ratio (MHR) can be used as potential markers of systemic inflammatory state, and significant increases in NLR have been reported in patients with $\mathrm{PD}^{[9-11]}$. However, $\mathrm{PD}$ is a heterogeneous disease with rapidly and slowly progressive forms.Although there are many treatment measures for this disease,progressive neurodegeneration and the increase of accompanying symptoms still impose a serious burden on the society ${ }^{[12]}$.At present, little is known about the relationship between NLR, NHR and MHR and the course and severity of PD.Therefore, it is of great significance to study the relationship between inflammatory factors and PD for prevention and treatment of PD.

\section{Methods}

2.1 Study population A retrospective analysis was performed on 198 patients hospitalized in the Department of Neurology of Qinghai Provincial People's Hospital from March 2017 to March 2020, including 100 males (50.5\%) and 98 females (49.5\%),ranging from 36 years old to 88 years old and 55.8 $\sim 76$ years old $(65.3 \pm 12.6$ years old).PD patients $(n=101)$ and control group $(n=97)$.According to the length of the disease cycle,PD patients were divided into three groups: $<6$ years $(n=64), 6-10 y e a r s(n=23)$ and $>10$ years $(n=14)$.According to Hoehn and Yahr classification, the disease severity of patients after treatment was divided into $1 \sim 2.5$ as early stage $(n=55)$. Grades $3 \sim 5$ are divided into two groups $(n=$ 46).Inclusion criteria:(1)Integral material clinical and laboratory examination data of patients;(2)Met the diagnostic criteria established by the International Parkinson's Disease and Movement Disorders Association ${ }^{[13]}$.The exclusion criteria were as follows:(1) the presence of signs of acute infection;(2)Acute cardiovascular disease; (3) Diabetes,hypothyroidism,tumor and blood immune system diseases;(4)Severe liver and kidney injuries.The study was approved by the ethics committee of Qinghai Provincial People's Hospital.

2.2 Blood methods Collect the list the basic information such as age,gender,research objects are all within 24h after admission morning fasting blood extract, white blood cells, neutrophils, monocytes and lymphocytes, Hb,HDL-C,LDL-C,uric acid,such as laboratory assay index, further calculate the mononuclear cells and high-density lipoprotein cholesterol (HDL-C) ratio (MHR), neutrophils ratio and high density lipoprotein cholesterol(NHR) and the ratio of neutrophils and lymphocytes (NLR).Beckman AU5800 automatic biochemical analyzer was used to measure HDL-C(selective inhibition method),LDL$\mathrm{C}$ (surfactant clearance method) and uric acid (uricase method). The blood count was measured using the Sysmex XN-1000 analyzer (Sysmex,Kobe, Japan) to detect white blood cell,neutrophils,monocytes,lymphocytes, $\mathrm{Hb}$ and other laboratory indicators.

2.3 Statistical analyses SPSS25.0 software (Chicago,IL,USA) was used. The measurement data were tested by S-W normality test, and the normal distribution was expressed as mean \pm standard deviation(MEAN $\pm S D$ ). T-test or one-way analysis of variance (ANOVA) were used, the non-normal distribution was expressed as median (quartile), Wilcoxon rank sum test was used, and chi-square test was used for enumeration data. To study the influencing factors of PD, univariate analysis was first 
performed, and variables with significant univariate screening $(P<0.05)$ were included in the multivariate logistic regression model with PD as the dependent variable for risk factor analysis. The ROC curve was used to analyze the factors affecting PD and judge its diagnostic value.Spearman correlation analysis was used for the diseased samples. In this analysis, $P<0.05$ was considered statistically significant.

\section{Results}

3.1 Comparison of baseline demographic characteristics and laboratory indicators between the two groups In the PD group $(n=101)$, there were 51 males $(51 \%)$ with an age of $67(55.5 \sim 75.5)$. In the control group $(n=97), 49$ males (49\%) aged $67(55.5 \sim 76)$ showed no statistically significant difference in gender and age between the two groups $(P>0.05)$, indicating comparability. White blood cells, monocytes, uric acid,TG, HDL-C and MHR had no significant difference between the two groups. The levels of neutrophils,NHR and NLR in the PD group were significantly higher than those in the control group,while the levels of lymphocytes, LDL-C and hemoglobin in the PD group were significantly lower than those in the control group, with statistically significant differences $(P<0.05, P<0.01$, Table 1$)$.

3.2 Risk factors analysis of PD patients Taking PD as the dependent variable,univariate analysis showed that the related factors of PD were Hb,TC,LDL-C,NHR and NLR as independent variables(because of the collinearity of NHR and NLR with neutrophils and lymphocytes, neutrophils and lymphocytes were not included in the multi-factor analysis). Multi-factor logistics regression analysis showed that $\mathrm{NHR}(P=$ $0.046, \mathrm{OR}=1.456>1)$ and $\mathrm{MHR}(P=0.016, \mathrm{OR}=1.663>1)$ were risk factors for PD patients, while $\mathrm{Hb}, \mathrm{TC}$ and LDL-C could not significantly affect whether patients had PD.The results were plotted as forest map $(P<0.05$,Fig. 1).

3.3 Difference analysis of PD patients with different onset cycles According to the duration of disease of PD patients, pair comparisons were carried out among 3 sub-groups of $<6$ years, $6-10$ years and $>10$ years. The results showed that WBC,neutrophil,TG and NHR with the onset period of more than 10 years were significantly lower than those of PD patients with the onset period of less than 6 years $(P<0.05)$. Please refer to the box diagram (Fig. 2) for the specific differences.

3.4 Difference analysis of PD patients with different severity Comparison of patients with early and middle-advanced stage PD showed no statistically significant differences in gender,WBC, Neutrophil, lymphocyte, monocyte,Hb, UA, TC,TG or LDL-C. However, there were significant differences in age,HDLC,MHR and NHR $(P<0.05)$, among which,the levels of year, MHR and NHR in early stage patients were significantly lower than those in advanced stage patients. HDL-C levels were significantly higher in early stage patients than in advanced stage patients. The above differences are drawn into a box diagram (Fig. 3).

3.5 ROC curve analysis ROC curve analysis was performed on NHR and NLR values to evaluate PD.For $\mathrm{NHR}$,the AUC was 0.648 (95\% Cl:0.572 0.724, $P=0.0003$ ), the best cut-off value was $3.104 \times 10^{9} / \mathrm{mmol}$, and the sensitivity\% was $46.53 \%$. Specificity $\%: 76.29 \%$. The AUC of NLR was 
$0.718(95 \% \mathrm{Cl}: 0.646 \sim 0.790, P<0.0001)$, and its optimal critical value was $1.939 \times 10^{9} / \mathrm{mmol}$, sensitivity\%:70.34\%.Specificity \%:69.17\% (Fig. 4).

3.6 Spearman analysis Since there were significant differences between NHR and MHR and the onset cycle and Hoehn-Yahr classification in PD patients,further correlation analysis showed that there was a significant negative correlation between NHR and the onset period, with a correlation coefficient of $-0.22, P=0.027<0.05$. The correlation with Hoehn-Yahr classification was not significant, $P>0.05$.MHR and course of disease and Hoehn-Yahr were not significant (Table 2). 
Table 1

Characteristics of PD patients compared with healthy controls

\begin{tabular}{|c|c|c|c|c|}
\hline & Controls $(n=97)$ & $P D(n=101)$ & $x^{2} / Z / T$ & $P$ \\
\hline Gender (male-\%) & $49(49 \%)$ & $51(51 \%)$ & $<0.001$ & 0.998 \\
\hline Age(year) & $67(55.5 \sim 76)$ & $67(55.5 \sim 75.5)$ & -0.148 & 0.883 \\
\hline $\mathrm{WBC}\left(\times 10^{9} / \mathrm{L}\right)$ & $5.11(4.66 \sim 5.88)$ & $5.49(4.62 \sim 6.4)$ & -1.536 & 0.125 \\
\hline Neutrophil $\left(\times 10^{9} / \mathrm{L}\right)$ & $2.86(2.3 \sim 3.42)$ & $3.36(2.86 \sim 4.21)$ & -4.256 & $<0.001$ \\
\hline Lymphocyte $\left(\times 10^{9} / \mathrm{L}\right)$ & $1.75(1.39 \sim 2.11)$ & $1.51(1.21 \sim 1.85)$ & -3.27 & 0.001 \\
\hline Monocyte $\left(\times 10^{9} / \mathrm{L}\right)$ & $0.35(0.29 \sim 0.42)$ & $0.35(0.31 \sim 0.46)$ & -1.091 & 0.275 \\
\hline $\mathrm{Hb}\left(\times 10^{9} / \mathrm{L}\right)$ & $155.32 \pm 18.5$ & $149.65 \pm 17.76$ & 2.199 & 0.029 \\
\hline $\mathrm{UA}(\mu \mathrm{mol} / \mathrm{L})$ & $312.12 \pm 66.16$ & $305.27 \pm 79.65$ & 0.658 & 0.512 \\
\hline $\mathrm{TC}(\mathrm{mmol} / \mathrm{L})$ & $4.8(4.03 \sim 5.37)$ & $4.15(3.74 \sim 4.9)$ & -3.441 & 0.001 \\
\hline $\mathrm{TG}(\mathrm{mmol} / \mathrm{L})$ & $1.3(0.97 \sim 1.8)$ & $1.24(0.87 \sim 1.83)$ & -0.758 & 0.448 \\
\hline $\mathrm{HDL}-\mathrm{C}(\mathrm{mmol} / \mathrm{L})$ & $1.19(1.01 \sim 1.35)$ & $1.18(1.01 \sim 1.37)$ & -0.483 & 0.629 \\
\hline LDL-C(mmol/L) & $2.81(2.37 \sim 3.32)$ & $2.42(1.95 \sim 3)$ & -3.244 & 0.001 \\
\hline $\operatorname{MHR}\left(\times 10^{9} / \mathrm{mmol}\right)$ & $0.29(0.24 \sim 0.37)$ & $0.31(0.24 \sim 0.43)$ & -1.186 & 0.236 \\
\hline $\mathrm{NHR}\left(\times 10^{9} / \mathrm{mmol}\right)$ & $2.42(1.97 \sim 3.05)$ & $2.81(2.25 \sim 3.84)$ & -3.592 & $<0.001$ \\
\hline $\operatorname{NLR}\left(\times 10^{9} / \mathrm{mmol}\right)$ & $1.61(1.25 \sim 2.14)$ & $2.22(1.77 \sim 2.93)$ & -5.293 & $<0.001$ \\
\hline \multicolumn{5}{|c|}{$\begin{array}{l}\text { Abbreviations:PD,Parkinson's disease;WBC, white blood cell;UA,Uric Acid;TC,total } \\
\text { cholesterol;Hb,Hemoglobin; }\end{array}$} \\
\hline \multicolumn{5}{|c|}{$\begin{array}{l}\text { TG,triglyceride;HDL-C,high-densitylipoprotein;LDL-C,low-density lipoprotein;MHR,monocyte-to-HDL } \\
\text { ratio; }\end{array}$} \\
\hline \multicolumn{5}{|c|}{ NHR,neutrophil-to-HDL ratio;NLR,neutrophil-lymphocyte ratio. } \\
\hline
\end{tabular}


Table 2

Correlation analysis of NHR and MHR with the course and Hoehn-Yahr classification

\begin{tabular}{|lllll|}
\hline & NHR & MHR & course of disease & Hoehn-Yahr \\
\hline NHR & 1 & & & \\
\hline MHR & $.687^{* *}$ & 1 & & \\
\hline course of disease & $-0.22^{*}$ & -0.021 & 1 & \\
\hline Hoehn-Yahr & 0.076 & 0.17 & $.412^{\star *}$ & 1 \\
\hline$*$ At the level of 0.05 (double-tailed),Significant correlation. & \\
\hline$*$ At the level of 0.01 (double-tailed),Significant correlation. \\
\hline
\end{tabular}

\section{Discussion}

The relationship between the levels of NHR, NLR and NHR in PD patients and the course and severity of disease was discussed. The main findings are as follows:(1) NHR and NLR were significantly correlated with PD. (2) With the progression of disease, NHR level decreased significantly. The more advanced PD patients are, the more NHR and MHR are, while there is no significant difference between NLR onset cycle and Hoehn-Yahr classification.(3) ROC curve analysis showed that AUC of NLR was greater than NHR (4) In Spearman analysis,and NHR was negatively correlated with disease course.Based on the above results, we speculated that NLR has a higher predictive value for PD, while NHR may be associated with chronic inflammation in PD patients.

Previous studies have shown that neutrophils have a strong ability to pass through any blood vessel wall and epithelial cells,recruit,activate, and regulate the transport of different leukocyte populations in tissues, and promote the body's inflammatory response through the mutual regulation of chemokines expressed and released by the body ${ }^{[14-15]}$. Our study showed that the level of neutrophils was significantly higher than that of the healthy control group, which also confirmed its promoting effect on the onset of PD to some extent.Not only the relationship between inflammation and PD disease and progression has been mentioned ${ }^{[4-6]}$,moreover,PD has a strong correlation with abnormal lipid metabolism ${ }^{[16]}$. However,the relationship between the level of HDL-C in peripheral blood and PD has not been determined.Compared with the control group, plasma HDL-C decreased significantly ${ }^{[17-18]}$ or was irrelevant ${ }^{[6,19-20]}$. This study found that HDL-C increased gradually with the extension of PD duration,which was consistent with previous studies ${ }^{[21]}$.The reasons for consideration are as follows: (1)Smoking has been proved to be negatively correlated with the prevalence rate of PD,and the decrease of smoking rate in PD patients may lead to the decrease of cardiovascular disease ${ }^{[22]}$. At the same time, one study found that smokers had lower HDL-C levels ${ }^{[11]}$ (2)Levodopa is the standard therapy for Parkinson's disease.Studies have reported that levodopa can reduce blood lipid level,but with the 
increasing course of the disease, it is often necessary to reduce the dose of levodopa to avoid motor complications ${ }^{[23]}$.Other reports have shown that PD patients have a cardiometabolic protective effect(3)Other reports have shown that PD patients have a cardiometabolic protective effect ${ }^{[24]}$, while $\mathrm{HDL}-\mathrm{C}$ has been recognized as a protective factor for cardiovascular disease, it is not difficult to understand that HDL-C levels rise during the course of the disease.Neutrophils were "closely related" to HDL-C.HDL-C can not only inhibit the expression of neutrophil active activators (PMA and fMLP), but also inhibit its activation,adhesion, diffusion and migration ${ }^{[25]}$.This is consistent with the findings of this study that neutrophils show a downward trend with the increase of disease course.Certainly, a meta-analysis ${ }^{[26]}$ shows that non-steroidal anti-inflammatory drugs (NSAIDs) are effective in the prevention and treatment of PD patients, and may also lead to decrease of neutrophils. Interestingly,the study concluded that HDL-C levels were significantly lower in patients with advanced PD than in patients with earlier stages.HDL-C plays an important role in the brain under both physiological and pathological conditions. Treatment with enhanced HDL-C function is beneficial to both the peripheral and central nervous systems ${ }^{[27]}$.ApoA1 exists in cerebrospinal fluid and is the most important structural protein component of HDL-C. Recent studies have reported that higher ApoA1 level can effectively delay the dopaminergic neurodegeneration in PD patients,thus reducing the severity of the disease ${ }^{[17]}$. Therefore, it is particularly important to pay attention to HDL-C in PD patients.

Infiltrating adaptive immune cells play an important role in neuroinflammatory diseases and neurodegenerative diseases ${ }^{[2]}$. The formation of Louis nucleosome is a characteristic pathological change in PD patients, which is mainly composed of -synuclein (-SYn). In an animal experiment, $T$ cell infiltration (e.g.,CD3 ${ }^{+}, \mathrm{CD}^{+}{ }^{+} \mathrm{T}$ cells) was detected in the neocortex, hippocampus, and striatum perivascular and parenchyma of -SYN mice.Immunoregulatory mediators and/or lack of effective mechanisms of dissolution led to a pro-inflammatory environment. In addition, T cell interaction with glial cells further enhanced inflammatory activity ${ }^{[5,28]}$. The results of this study indicated that the lymphocyte level in peripheral blood was significantly lower than that of the control group,which was considered to be related to the recruitment of lymphocytes into the brain parenchyma,leading to the imbalance of lymphocytes and the reduction of $\mathrm{CD}^{+}$and $\mathrm{CD} 4^{+}$lymphocyte subsets in $\mathrm{PD}$ patients,especially $\mathrm{CD}^{+}{ }^{+}$, which was the most abundant type of lymphocytes ${ }^{[29]}$.

In conclusion, it is not difficult to conclude that immune inflammatory reaction and dyslipidemia are important factors to promote the occurrence and progression of PD.NHR and NLR were determined by the ratio of neutrophils to HDL-C and neutrophils to lymphocytes, respectively,so it was speculated that the two indexes were related to PD.They are not only simple and fast,but also integrate the information between factors. Compared with a single indicator, they have advantages and reflect the complementary relationship between different pathways, so as to more accurately reflect the inflammatory changes of PD.Therefore,NHR and NLR were considered to be closely related to inflammatory activities and lipid changes.Previous studies have reported that the interaction between increased neutrophils and decreased HDL-C leads to increased NHR level and increased risk of acute myocardial infarction in the elderly ${ }^{[30]}$.In 
an analysis of NHR and prognosis after intravenous thrombolysis in acute ischemic stroke ${ }^{[10]}$, With the aggravation of nerve injury,NHR level gradually increased,and $25 \%$ of patients with high NHR level developed severe neurological impairment.PD is a chronic degenerative disease, with the greatest neurological burden between 80 and 89 years old ${ }^{[1]}$.Our results showed that NHR decreased significantly with the progression of the disease, and NHR increased with the advanced stage of PD.NHR is negatively correlated with course of disease and can be an effective predictor of long-term clinical outcomes.NLR has been widely studied, except for being reported as an inflammatory marker for cardiovascular and cerebrovascular diseases and tumors ${ }^{[31-33]}, \mathrm{NLR}$ is also associated with PD, but not with different clinical subtypes,considering that dopamine replacement therapy can change the state of peripheral inflammation through its metabolites, other reports have shown that NLR is positively correlated with the increase of homocysteine caused by the decomposition of levodopa ${ }^{[6,34-35]}$. Our study showed that NHR and NLR were closely correlated in terms of influencing the incidence and prediction of PD, but the latter was better. In conclusion, it is suggested that the comprehensive application of NHR and NLR in different stages of PD patients can help improve the diagnosis,treatment and prognosis of PD patients in subsequent clinical work.

However,there are still some limitations in the research process. Firstly, due to retrospective analysis, the effect of NHR and NLR levels on the prognosis of PD patients was limited.Secondly, the sample size involved is relatively small and comes from the same hospital, which makes the results lack of universality.Finally, the specific influence mechanism of NHR and NLR on the occurrence and development of PD is still unknown,and more studies are needed to improve it.

\section{Declarations}

\section{Ethical Approval and Consent to participate}

We further confirm that any aspect of the work covered in this manuscript that involved human patients was conducted with the ethical approval of all relevant bodies.

\section{Consent for publication}

All authors and participants have given their consent for publication of this article in Lipids in Health and Disease.

\section{Availability of data and material}

The datasets used and/or analyzed during the current study are available from the corresponding author on reasonable request.

\section{Competing interests}

The authors declare that they have no known competing financial interests or personal relationships that could have appeared to influence the work reported in this paper. 


\section{Funding}

This study was supported by grants from"Clinical medical reserach center project of Qinghai Province"(Grant\#2017-SF-L1) and program funds.

\section{Authors' contributions}

In this study,ZL,SHZ,and QLF did the study design, statistical analyses and results interpretation. YCL and WFY participated as analyzing and resolving difficulties of analytic strategies and results discussion. Finally,QLF functioned as final reviewer and corresponding author. All authors read and approved the final manuscript.

\section{Acknowledgements}

We would like to express our gratitude to all those who exert their effects in achieving this study.

\section{References}

1. GBD. 2016 Neurology Collaborators,Global, regional, and national burden of neurological disorders, 1990-2016: a systematic analysis for the Global Burden of Disease Study 2016.[J].Lancet Neurol, 2019, 18: 459-480.

2. Kline Elizabeth M, Houser Madelyn C, Herrick Mary K, et al. Genetic and Environmental Factors in Parkinson's Disease Converge on Immune Function and Inflammation.[J].Mov Disord, 2020, undefined: undefined.

3. Joers Valerie,Masilamoni Gunasingh,Kempf Doty. et al. Microglia, inflammation and gut microbiota responses in a progressive monkey model of Parkinson's disease: A case series.[. J]Neurobiol Dis. 2020;144:105027.

4. YuChiun-Chieh,Chen H-L,Chen Meng-Hsiang, et al. Vascular Inflammation Is a Risk Factor Associated with Brain Atrophy and Disease Severity in Parkinson's Disease: A Case-Control Study.[J].Oxid Med Cell Longev, 2020, 2020: 2591248.

5. Pajares Marta I, Rojo Ana,Manda Gina, et al. Inflammation in Parkinson's Disease: Mechanisms and Therapeutic Implications.[J].Cells, 2020, 9: undefined.

6. Umehara Tadashi,Oka Hisayoshi,Nakahara Atsuo. et al. Differential leukocyte count is associated with clinical phenotype in Parkinson's disease.[. J]J Neurol Sci. 2020;409:116638.

7. Nissen Sara Konstantin,Shrivastava Kalpana,Schulte Claudia. et al. Alterations in Blood Monocyte Functions in Parkinson's Disease. [J]Mov Disord. 2019;34:1711-21.

8. Chiesa Scott T,Charakida Marietta,McLoughlin Eve, et al. Elevated high-density lipoprotein in adolescents with Type 1 diabetes is associated with endothelial dysfunction in the presence of systemic inflammation.[J].Eur Heart J, 2019, 40: 3559-3566. 
9. Akıl, Eşref,Bulut Aslıhan,Kaplan İbrahim et al. The increase of carcinoembryonic antigen (CEA), highsensitivity C-reactive protein, and neutrophil/lymphocyte ratio in Parkinson's disease.[J].Neurol Sci, 2015, 36: 423-428.

10. Chen Guangyong,Yang Naiping,Ren Junli. et al. Neutrophil Counts to High-Density Lipoprotein Cholesterol Ratio: a Potential Predictor of Prognosis in Acute Ischemic Stroke Patients. After Intravenous Thrombolysis[J]Neurotox Res. 2020;38:1001-9.

11. Yılmaz, Mücahid. Kayançiçek Hidayet,A New Inflammatory Marker: Elevated Monocyte to HDL Cholesterol Ratio Associated with Smoking.[. J]J Clin Med. 2018;7:undefined.

12. Armstrong Melissa J, Okun Michael S. Diagnosis and Treatment of Parkinson Disease. A Review[J]JAMA. 2020;323:548-60.

13. Berardelli A, Wenning GK, Antonini A, et al. EFNS/MDS-ES recommendations for the diagnosis of Parkinson's disease[J]. Eur J Neurol. 2013;20(1):16-34.

14. Ley Klaus,Hoffman Hal M,Kubes Paul et al. Neutrophils: New insights open questions[J]Sci Immunol, 2018, 3: undefined.

15. Tecchio Cristina. Cassatella Marco A,Neutrophil-derived chemokines on the road to immunity.[. J]Semin Immunol. 2016;28:119-28.

16. Xicoy, Helena. Wieringa Bé,Martens Gerard J M,The Role of Lipids in Parkinson's. Disease[J]Cells. 2019;8:undefined.

17. Swanson Christine R,Berlyand Yosef,Xie Sharon X et al. Plasma apolipoprotein A1 associates with age at onset and motor severity in early Parkinson's disease patients. [J].Mov Disord, 2015, 30: 1648-1656.

18. Guo Xiaoyan,Song Wei,Chen Ke. et al. The serum lipid profile of Parkinson's disease patients: a study from China.[. J]Int J Neurosci. 2015;125:838-44.

19. Thaler Avner,Shenhar-Tsarfaty Shani,Shaked Yanay. et al. Metabolic syndrome does not influence the phenotype of LRRK2 and GBA related Parkinson's disease.[. J]Sci Rep. 2020;10:9329.

20. Jin Hong,Gu Han-Ying,Mao Cheng-Jie. et al. Association of inflammatory factors and aging in Parkinson's disease.[. J]Neurosci Lett. 2020;736:135259.

21. Cassani Erica,Cereda Emanuele,Barichella Michela. et al. Cardiometabolic factors and disease duration in patients with Parkinson's disease.[J]. Nutrition. 2013;29:1331-5.

22. Alves Guido,Kurz Martin,Lie. Stein A. et al. Cigarette smoking in Parkinson's disease: influence on disease progression.[J]Mov Disord, 2004, 19: 1087-92.

23. Scigliano Giulio,Ronchetti Gabriele,Girotti Floriano. et al. Sympathetic modulation by levodopa reduces vascular risk factors in Parkinson disease.[. J]Parkinsonism Relat Disord. 2009;15:138-43.

24. Morley James F. Duda John E,Parkinson's disease and the risk of cerebrovascular pathology.[. J]Mov Disord. 2012;27:1471-2.

25. Murphy Andrew J, Woollard Kevin J, Suhartoyo Andreas. et al. Neutrophil activation is attenuated by high-density lipoprotein and apolipoprotein A-I in in vitro and in vivo models of inflammation.[J]. 
Arterioscler Thromb Vasc Biol. 2011;31:1333-41.

26. Gagne Joshua J,Power Melinda C,Anti-inflammatory drugs and risk of Parkinson disease: a metaanalysis.[J].Neurology, 2010, 74: 995-1002.

27. Hottman David A,Chernick Dustin,Cheng Shaowu et al. HDL and cognition in neurodegenerative disorders.[J].Neurobiol Dis, 2014, null: 22-36.

28. Iba Michiyo,Kim Changyoun,Sallin Michelle. et al. Neuroinflammation is associated with infiltration of T cells in Lewy body disease and a-synuclein transgenic models.[. J]J Neuroinflammation. 2020;17:214.

29. Jiang Sen,Gao Hua,Luo Qin. et al. The correlation of lymphocyte subsets, natural killer cell, and Parkinson's disease: a meta-analysis.[. J]Neurol Sci. 2017;38:1373-80.

30. Huang Jia-Bao,ChenYu-Si,Ji Hong-Yan et al. Neutrophil to high-density lipoprotein ratio has a superior prognostic value in elderly patients with acute myocardial infarction: a comparison study. [J].Lipids Health Dis, 2020, 19: 59.

31. Nam Ki-Woong,Kwon Hyung-Min,Jeong Han-Yeong et al. High neutrophil to lymphocyte ratios predict intracranial atherosclerosis in a healthy population.[J].Atherosclerosis, 2018, 269: 117-121.

32. Wang Lu,Song, Quhong,Wang, Changyi. et al. Neutrophil to lymphocyte ratio predicts poor outcomes after acute ischemic stroke: A cohort study and systematic review.[. J]J Neurol Sci. 2019;406:116445.

33. Galvano Antonio,Peri Marta,Guarini Aurelia Ada. et al. Analysis of systemic inflammatory biomarkers in neuroendocrine carcinomas of the lung: prognostic and predictive significance of $N L R, L D H, A L I$, and LIPI score.[J]. Ther Adv Med Oncol. 2020;12:1758835920942378.

34. Ataç Uçar Ceyla,Gökçe Çokal Burcu,Ünal Artık Hanzade Aybüke et al. Comparison of neutrophillymphocyte ratio (NLR) in Parkinson's disease subtypes.[J].Neurol Sci, 2017, 38: 287- 293.

35. Gang, Li. Yanyan Zhang,Increased neutrophil to lymphocyte ratio in persons suffering from hypertension with hyperhomocysteinemia.[. J]Hypertens Res. 2016;39:606 - 11.

\section{Figures}




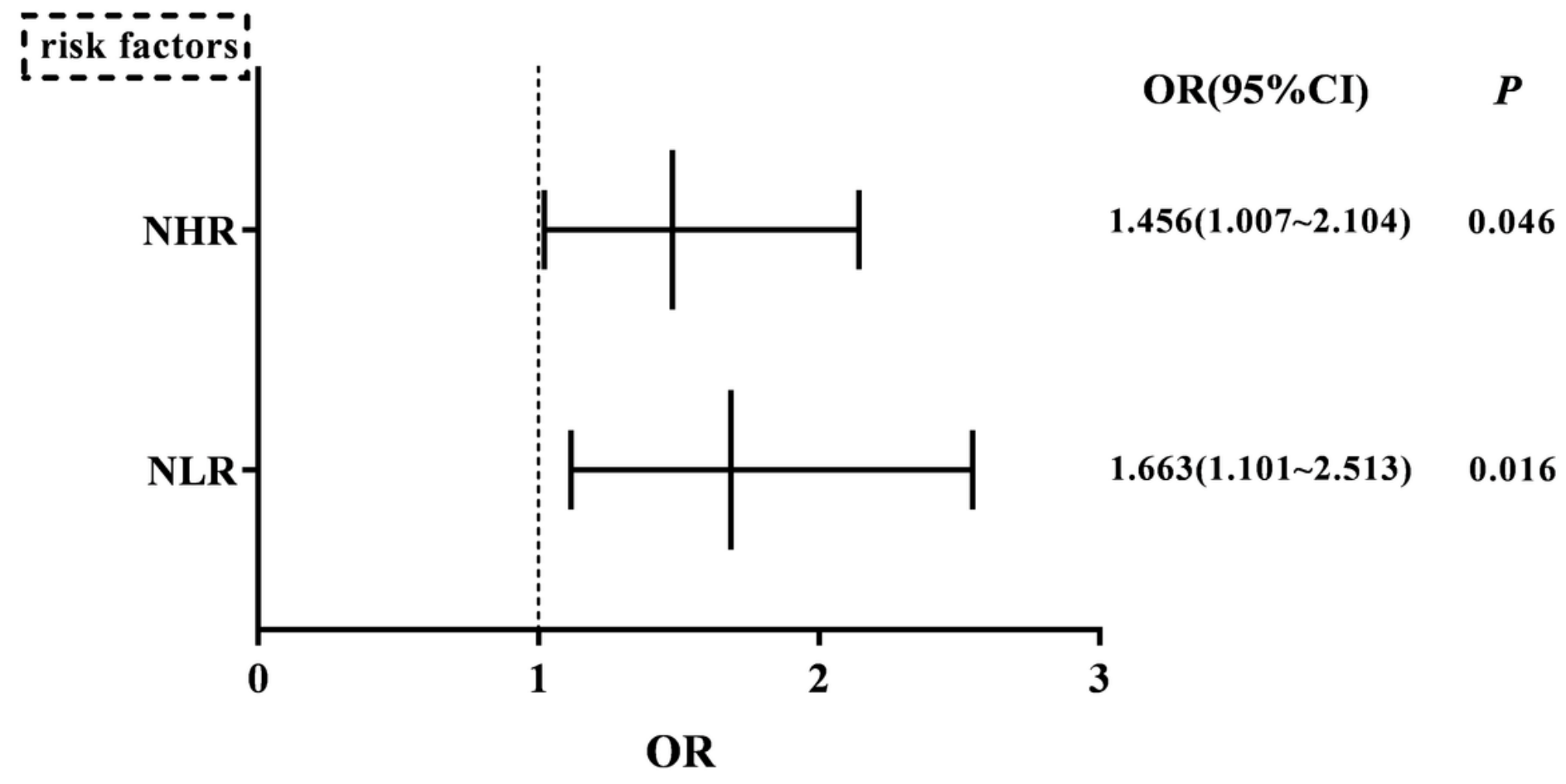

Figure 1

Analysis of risk factors in PD patients 

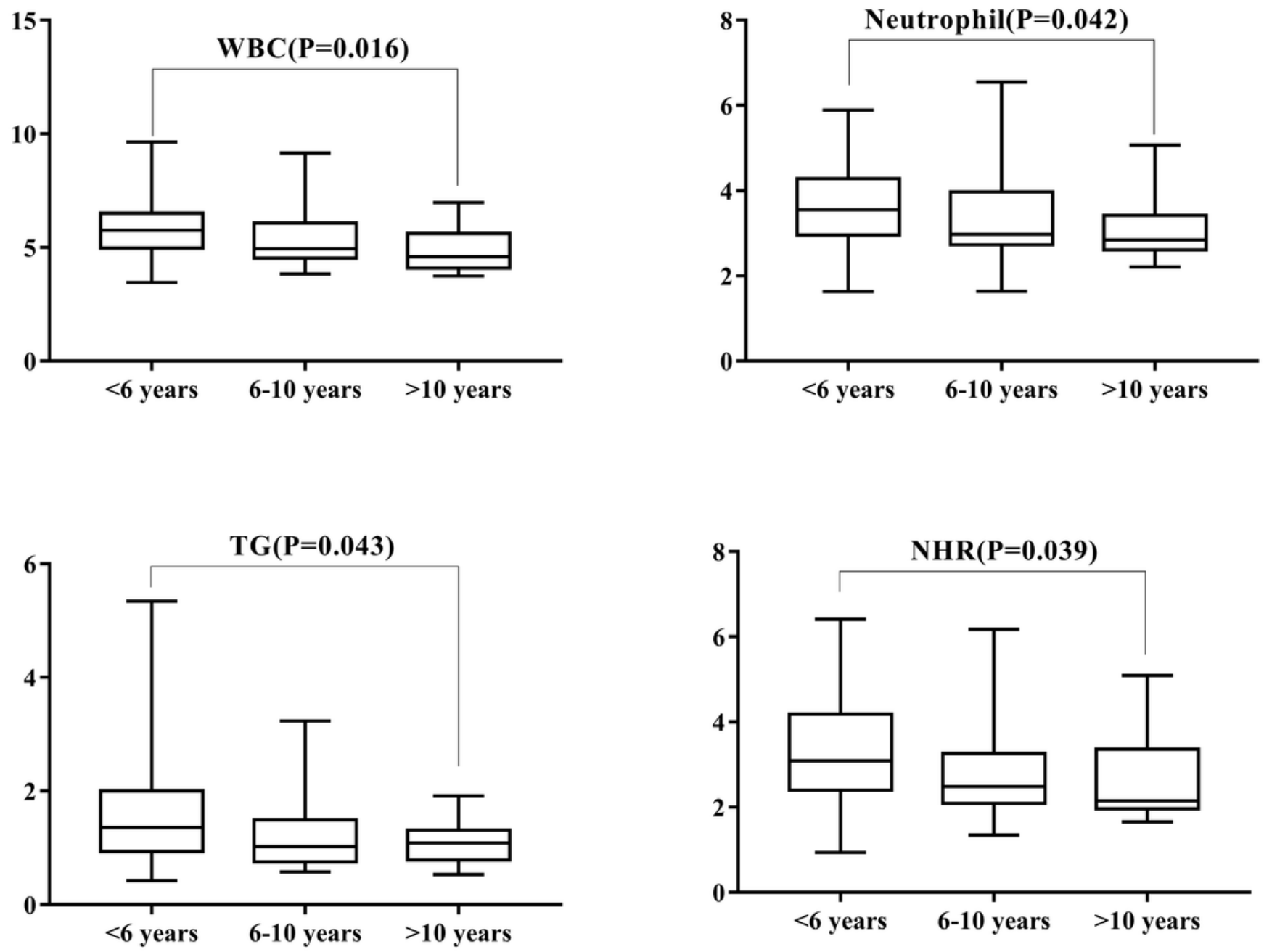

Figure 2

WBC, neutrophil, TG and NHR of PD patients with different course of disease 

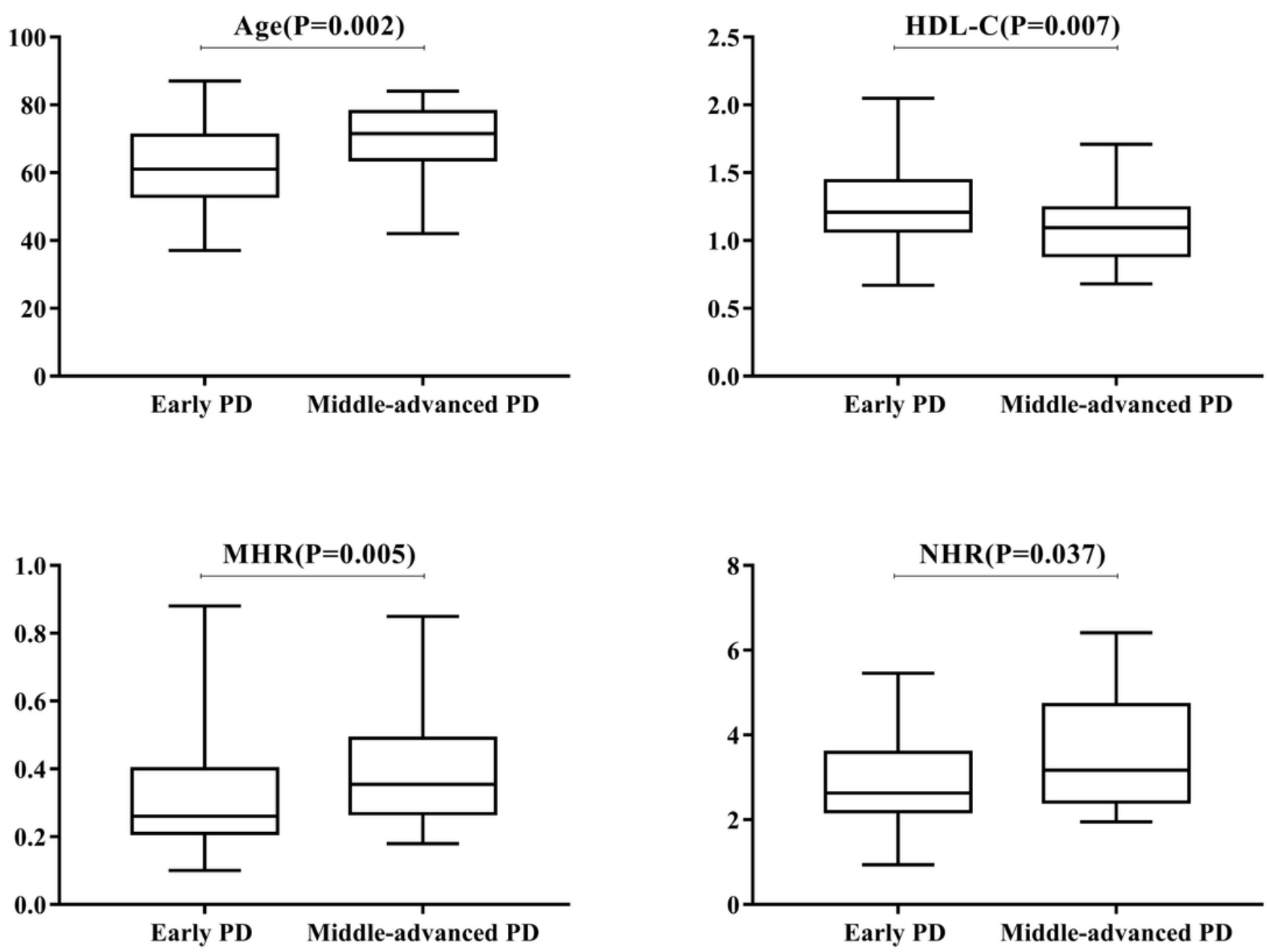

Figure 3

Differences in age, HDL-C,MHR and NHR between patients with early and middle-advanced Parkinson's disease 


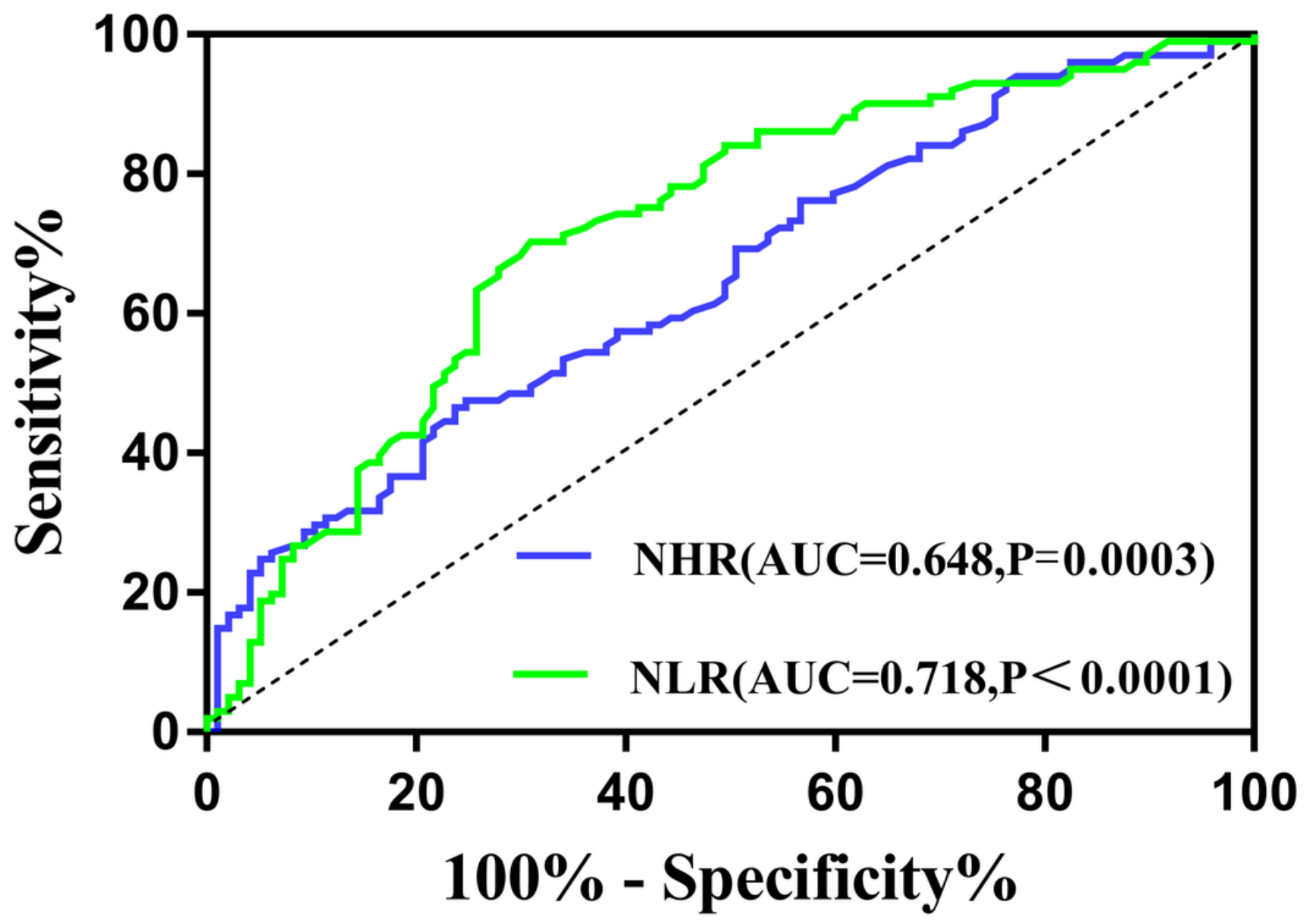

Figure 4

ROC curve analysis of PD patients with NHR and NLR 